Cahiers de Narratologie

Analyse et théorie narratives

22 | 2012

Voix off et narration cinématographique. Second volet

\title{
Voix-off et voix(e) narrative : la conscience entre les lignes dans l'œuvre de Arnaud Desplechin
}

\section{Marie-Anne Lieb}

\section{(2) OpenEdition}

\section{Journals}

Édition électronique

URL : http://journals.openedition.org/narratologie/6519

DOI : 10.4000/narratologie.6519

ISSN : $1765-307 X$

Éditeur

LIRCES

Référence électronique

Marie-Anne Lieb, «Voix-off et voix(e) narrative : la conscience entre les lignes dans l'œuvre de Arnaud Desplechin», Cahiers de Narratologie [En ligne], 22 | 2012, mis en ligne le 20 juillet 2012, consulté le 30 avril 2019. URL : http://journals.openedition.org/narratologie/6519; DOI : 10.4000/narratologie.6519

Ce document a été généré automatiquement le 30 avril 2019

Article L.111-1 du Code de la propriété intellectuelle. 


\title{
Voix-off et voix(e) narrative : la conscience entre les lignes dans l'œuvre de Arnaud Desplechin
}

\author{
Marie-Anne Lieb
}

1 D’essence romanesque, la filmographie de Desplechin décline des récits introspectifs : aventure spirituelle selon Esther Kahn (2000), marivaudage dans Comment je me suis disputé (ma vie sexuelle) en 1996, thrillers métaphysiques pour La Sentinelle (1991) et Léo en jouant Dans la compagnie des hommes (2003) ou bien quête existentielle avec La Vie des morts (1990), teintés de mélo et de comédie pour Rois et Reine (2005) et Un conte de Noël (2007). Fruits improbables d'un éclatement des trames spatio-temporelles et d'une continuité digne du nappé barthésien, les récits chez Desplechin cherchent à traduire la complexité humaine. Les personnages placent le langage et le commentaire en leur centre comme un aveu d'une maitrise illusoire de l'existence. Par ailleurs, telle une introduction à l'utilisation des voix par le cinéaste (narratives, actorielles, dialogiques et off), cet état de fait permet aussi de questionner les niveaux de réalité et de temporalité, car l'oralité multiple dans l'œuvre de Desplechin apparaît comme un outil de cohésion tandis que les images et leur montage nourrissent l'éclatement. La voix-off se travestit à la troisième personne du singulier partagée entre narrateur et acteur sous forme de narration explicative dans $L a$ Sentinelle et Comment je me suis disputé (ma vie sexuelle), puis à la première personne du singulier de l'acteur dans Léo en jouant Dans la compagnie des hommes et Rois et Reine, pour enfin laisser place à plusieurs voix (off et in) de personnages racontant les fondations de l'histoire familiale sous formes d'adresses à la caméra comme dans Un conte de Noël. De la voix-off à la voix(e) narrative, aux confins de la réalité et de la fiction, entre le soi et l'autre, on s'aperçoit que c'est la conscience qui s'exprime.

2 Nous savons que le discours narratoriel permet au cinéaste de mener son récit, tout en accentuant le jeu de la subjectivité et de l'objectivité entre les divers points de vue et le narrateur omniscient. Tout comme nous sommes sûrs qu'il amène le caractère littéraire dans la fiction cinématographique dès que l'impersonnalité $\mathrm{du}$ «il» est considérée. Présent dans quatre films chez Desplechin, le discours narratoriel est pris en charge par 
le cinéaste lui-même à l'exception du film Esther Kahn dans sa version anglaise originale puisque la voix est celle de Ramin Gray. Desplechin ouvre aussi le récit de Comment je me suis disputé (ma vie sexuelle) mais n'intervient plus qu'à des moments précis comme la description de l'a-intimité qui lie Paul à Nathan, le mimétisme inconscient qui unit les cousins Bob et Paul, l'aveu de sa liaison avec Sylvia ou bien encore lorsque Paul quitte Esther. Ce discours brosse leurs portraits puis énonce vite ce que les personnages peinent à formuler par eux-mêmes, engendrant autant une gêne d'avouer quelque chose qu'il est le signe d'une économie narrative. La voix-off dans plusieurs récits de Desplechin précède légèrement les images (overlapping) ou commente une action en étant synchrone avec elles alors que les sons sont inexistants ou diffus. Elle finit par se substituer de temps à autre à la « voix intérieure » du personnage.

3 S'agissant du récit d'Esther Kahn, la voix-off renforce son caractère romanesque, lui donnant une autre envergure. Récit adapté d'une nouvelle d'Arthur Symons, le spectateur est entrâné au sein d'une famille de tailleurs juifs londoniens grâce au discours narratoriel qui l'ouvre et le clôt. Une succession d'images répétées et montées en fondu enchaîné montre le caractère itératif de leur vie et accompagne le spectateur jusqu'à ce qu'il découvre Esther. Plus tard au cours de la visite médicale, la voix-off narratorielle s'attarde sur les grands yeux d'animaux endormis de l'héroïne tandis qu'elle recouvre à la toute fin les images de la première de Hedda Gabler, pièce du dramaturge Ibsen. Cette voixoff ne temporalise jamais le film et ne joue donc pas avec les temps grammaticaux qui annonceraient la subdivision du temps sur la ligne de l'histoire du personnage et sur celle de l'Histoire du Londres à la fin du XIXème.

Le personnage d'Esther est ancré dans l'immédiateté et semble n'avoir ni passé ni futur à l'inverse des films de Chris Marker dont le personnage est souvent présenté comme "ayant été » et "allant être ». Ainsi, le spectateur pénètre son monde grâce à la description progressive de ses émotions, de son hébétude ou de sa naissance en scène, la voix-off narratorielle s'infiltrant progressivement dans la tête du personnage qui apparaît de prime abord opaque, solitaire et inerte. Le contraste est d'autant plus grand qu'Esther ne maîtrise pas le verbe comme Paul, le héros de Comment je me suis disputé ( $m a$ vie sexuelle). Elle est plus intuitive que réflexive.

5 Le jeu entre objectivité et subjectivité n'intervient dans les récits de Desplechin qu'au travers de la structure narrative bâtie sur la contiguïté entre rupture et liaison mais également de la complexité du personnage à l'écran. En effet, à l'image de la narration chez Alain Resnais, l'histoire racontée échappe généralement à la simplicité du monde de l'enfance pour atteindre la complexité du monde adulte. Le monde n'est pas un, il est multiple. Aussi, dilater, condenser, monter, fragmenter, raccorder répond-il d'une autre manière à la logique de la discontinuité romanesque digne de celle de l'écrivain Novalis qui considère que l'art du roman exclut toute continuité car il doit être un édifice articulé dans chacun de ses périodes. Mais outre l'envie de Desplechin d'une narration illimitée, ces démultiplications supposent que la délinéarisation temporelle rende compte du morcellement psychique du personnage. Si Desplechin a souvent été narrateur, il dispose par la suite les voix en off de ses propres personnages de façon récurrente. Leur utilisation est relativement classique puis à chaque nouveau récit, leur fonction devient de plus en plus profonde et intrigante. Alors que le romanesque s'accorde aux quelques flash-back qui mettent en évidence cet aspect confus et fragmenté de toute existence, ceux-ci entrecoupent le récit premier en cassent le nappé et installent en conséquence un décalage entre vérité et mensonge, accentuant la dualité du personnage. Plus qu'une 
simple figure narrative digne de tout récit rétrospectif, c'est une façon habile de dédoubler les diverses strates du personnage rompu à se contempler et à s'étudier. La discordance qui en ressort révèle leur intériorité. Les récits rétrospectifs considérés ici ouvrent graduellement l'espace de la conscience. La discordance narrative de La Sentinelle indique-t-elle clairement que le flash-back s'amuse à créer un intervalle entre ce qui est entendu et ce qui est vu? Une séquence en particulier présente une alternance de plans au présent et ceux d'un souvenir au passé immédiat. Mathias, étudiant en médecine légale et malmené par son colocataire travaillant à la DGSE, soigne ce dernier d'une brûlure de cigarette au creux de la main. Desplechin choisit de monter cette séquence alors que la voix-off actorielle de Mathias recouvre les deux types d'images. Nous nous rendons vite compte que le héros aménage le réel car ses propos diffèrent d'une nappe temporelle à l'autre, du présent au passé, voire ne sont même pas pris en compte à l'image comme si ce qui se disait dans une des temporalités était occulté consciemment dans l'autre.

Poursuivons avec la séquence introductive de Comment je me suis disputé (ma vie sexuelle) qui insiste pertinemment et plus longuement sur cet aspect. Dès le premier plan sur le personnage principal, Paul endormi à son bureau, la voix-off du narrateur externe (Desplechin en l'occurrence) fait son portrait en quelques plans et quelques phrases. S'ensuit le générique qui décline des gravures et des images de type cinématographique sur une ville indéterminée nimbée de bleu. Nous repérons tout au plus des arches, des canaux et une rivière. Puis, une voix-off différente de la première couvre la fin du générique et diverses images dont celle en premier d'un petit garçon jouant sous la table d'un salon. Cette seconde voix-off (toujours non identifiée) poursuit son explication et précise que ce jour là, «mes cousins et moi, on jouait à Venise après avoir été visiter Bruges ».

7 Un plan à sa suite montre un autre jeune garçon attelé à son bureau. À la fin du récit dans lequel la voix-off raconte que, jeune, il avait tenté d'écrire «un récit vengeur à la Stevenson en tordant le cou à la vraie vie ", un plan s'ouvre sur Paul, adulte, allongé sur un divan chez son psychanalyste. Nous identifions enfin cette seconde voix-off, qui est celle de Paul adulte et désormais de nature actorielle. Le spectateur comprend ainsi que les images du générique sont celles de Bruges alors que le commentaire disait qu'ils avaient joué à Venise. En plus d'être l'illustration d'un souvenir d'enfance, ces images illustrent l'intervalle entre objectivité et subjectivité. Notons qu'une musique à la harpe renvoie à la mort, au rêve et à tout ce qui a trait aux esprits de manière générale. Aussi pouvons-nous demander si ce personnage n'émerge pas de sa propre tête, de sa propre imagination. D'autant que la séance avec son psychanalyste indique qu'il est «en chantier ", en voie de (re)construction comme le laissent entendre formellement et métaphoriquement les déplacements par les ouvriers de planches de bois, pour des travaux, dans une pièce annexe. D'autre part, au plan de Paul adulte endormi et réveillé par le bruit extérieur à son bureau, renchérit le premier plan du flash-back de Paul enfant en train d'écrire. L'un est tiré d'un songe par le bruit dans le couloir de la faculté quand l'autre s'invente un destin «à la Stevenson». Il faut aussi considérer, tout particulièrement à l'intérieur de ce flash-back, le premier petit garçon qui s'amuse à entrouvrir un rideau de velours bordeau faisant office de porte à une cabane enfantine et imaginaire. Le spectacle, la mise à distance et la représentation sont ainsi mis en évidence. Paul enfant tente de maîtriser son récit comme Paul adulte le fait en rapportant ce souvenir à son psychanalyste. La fin même de la séquence du flash-back sur le plan 
muet d'une réunion de famille creuse ce sillon. La voix-off de Paul commente et se rappelle que sa mère ayant trouvé ses débuts d'écrivain médiocres et vexants à leurs égards, entra dans une rage folle tandis que son père semblait dire à son fils par son regard: «tu vois pourquoi ma vie n'est pas un roman?», plan à l'appui sur ce regard. Cette dernière phrase souligne que face au réel, ici inconfortable, le personnage cherche à aménager son récit pour qu'il se rapproche le plus possible d'un roman jusqu'à ce que cette réécriture envahisse le récit premier. La mise à distance du réel par la voix-off permet au personnage de «tordre le cou à la vraie vie » et de s'inventer lui-même.

Au fur et à mesure de sa filmographie, Desplechin opère un effacement du discours narratoriel, celui-ci se réduisant à une phrase énoncée en guise de prologue. L'omniscience disparaît au profit du savoir d'un personnage unique. Figure de rétrécissement relativement traditionnelle, puisque le narrateur externe est une sorte de double de l'écrivain du roman ou de la nouvelle adaptée, et dès lors que le discours actoriel des personnages (la " voix intérieure ») prend le relais du discours narratoriel. De plus, la voix-off du narrateur acteur chez Desplechin est souvent le déclencheur de l'action et renvoie à l'autobiographie dont Rousseau se veut être le précurseur. Dès SaintAugustin, ce genre est apparu, ouvrant la voie à un exercice individualiste avec ses Confessions. Aussi, derrière cette illusion de confession de la part de certains personnages et une volonté avérée de maitriser son récit de vie, c'est un écho à ce pan de la littérature en insistant sur le motif du cloisonnement constamment présent chez ce cinéaste. Les personnages commentent le récit comme les héros truffaldiens de Tirez sur le pianiste (1960) ou L'Homme qui aimait les femmes (1977) et leurs points de vue organisent le tout. Le film Esther Kahn annonce clairement cette transition narrative. La voix-off narratorielle ouvre puis ferme le récit sobrement tandis que la dernière séquence laisse enfin entendre la voix en off de l'héroïne, dépourvue jusqu'alors de mots. En scène, elle interprète le personnage d'Hedda Gabler meurtri par les infidélités de son mari. Elle-même blessée par son ex-amant, Esther est dans un état second sur le plateau et son expérience théâtrale est filmée comme si elle jouait dans une bulle ouatée sans bruits diégétiques, ni dialogues audibles. Seule une musique extra-diégétique accompagne les plans sur les personnages, filmés de surcroît au ralenti et légèrement flous selon leurs déplacements dans le cadre. Puis, après la scène du suicide d'Hedda, le spectateur entend la voix en off d'Esther. En effet, lors des derniers plans du récit, nous la voyons quitter le théâtre, affronter son examant alors que sa voix-off décrit par delà les images, ses sensations, sa douleur à vouloir vivre, regrettant amèrement l'avoir souhaitée. Sa conscience est mise à nu. Cette transition narrative dans l'utilisation de la voix-off s'apparente sur le fond et la forme à celle qu'effectue Beckett dans son œuvre. En effet, c'est avec les récits Molloy, Malone meurt et l'Innommable que survient une rupture radicale dans son écriture et la littérature moderne. Le «je» remplace désormais le «il» pour tous les personnages. L'usage particulier du «je» par l'écrivain est une sorte de révolution littéraire. Alors que le plus souvent il est utilisé pour poser un caractère, donner du relief à l'existence d'une personne tout en délimitant son univers. Chez Beckett, le «je» est la source de toutes les voix et le point de départ d'une évasion en dehors de la réalité. Dire «je», c'est chercher à épuiser l'identité pour accéder à la vérité complexe du parler. À la distance entre un narrateur et ses personnages s'est substituée la multiplication des doubles intérieurs. Le monde romanesque beckettien se construit sur l'impossibilité de fixer un sens à l'existence, à laquelle seule l'errance avec les mots semble donner un contenu. 
Prenons l'exemple de Rois et Reine : la voix-off de Desplechin cite la légende de Léda et de Zeus en incipit sur le générique. L'ouverture du film présente un personnage féminin (Nora) qui descend d'un taxi sur les boulevards parisiens. Le plan suivant raccorde avec l'intérieur d'un appartement. Une alternance se met en place, tantôt avec des plans de Nora au présent du récit premier (taxi, galerie), tantôt à un temps postérieur, chez elle, face caméra, tandis que sa voix-off actorielle amorce le récit ainsi : "Je m'appelle Nora Cotterelle. J'ai trente-trois ans ». Répond-elle à une interview ? Nous pouvons le supposer car le dispositif est fixe et Nora assise bien en face même si son regard n'est pas toujours face caméra et s'adresse donc à un interlocuteur hors champ. Narratrice de sa vie, le spectateur apprend qu'elle est à la fois veuve, mère d'un fils de dix ans, qu'elle va se remarier avec Jean-Jacques et dirige une galerie d'art depuis six mois. Elle choisit une gravure pour son père (Léda et le cygne en lien avec l'incipit) puis l'action enchaîne sur le quai d'une gare où son futur mari l'attend. Son récit en voix-off se poursuit lors de son voyage en train vers Grenoble. Le discours actoriel de Nora s'arrête à son tour et ne reviendra que pour clore le récit à l'issue de la séquence de la visite au Musée de l'Homme par Ismaël et Elias. Un plan montre Elias courant rejoindre sa mère alors qu'en voix-off, Nora déclare : «J'ai aimé quatre hommes et j'en ai tué deux mais cela ne signifie rien. Je n'ai pas de regrets (...) Je suis enfin en paix ». Les adieux se font, puis on retrouve Nora chez elle, selon le même dispositif que dans le tout premier plan. Cette voix-off introduit par ailleurs la notion de « fonction réparatrice » de l'autobiographie comme il est d'usage de le penser à propos de l'écrit et qu'Un Conte de Noël illustre de bout en bout et de diverses façons. Après une commémoration au pied de la tombe de Joseph faite par son père, un personnage féminin raconte l'histoire de sa famille débutant par le cancer de son frère aîné Joseph, mort en bas âge et que les naissances d'une fille (Elizabeth) et de deux autres garçons (Henri «le fils inutile » et Yvan) n'ont pu sauver. Narratrice principale, la voix-off d'Elizabeth résonne tandis que les plans montrent un petit théâtre constitué de dessins enfantins et de silhouettes en papier en guise de marionnettes. Nous saurons plus tard que ce personnage est metteur en scène de théatre et cette ouverture devient à la fois une manière d'identifier l'univers du personnage, d'articuler la mise à distance du récit personnel que les personnages font de leur vie et enfin de saisir leur volonté à ordonner le récit. Conscience malheureuse du film, Elizabeth cherche à se comprendre et confirme la notion de réparation par la parole. Fil conducteur, elle agit également tel un filtre pour la notion de vraisemblance de toute l'histoire. Il est difficile de savoir vraiment si les accusations à l'encontre de son frère, qu'elle a banni, sont aussi graves ou bien si leurs parents ont accepté cet état de fait avec autant de facilité.

Concernant le caractère autobiographique, il est question ici d'une corrélation avec la narration réaliste du XIXème en littérature qui aimait recourir à la « voix intérieure ». Diderot l'attribuait à son personnage Jacques (Jacques le fataliste et son maître -1796) et instaurait une écriture romanesque moderne au sein de laquelle les niveaux de récit se chevauchaient, où les instances d'énonciation se multipliaient - les personnages devenant narrateurs - et où les digressions par rapport au thème central sans cesse annoncé et sans cesse différé (le récit des amours de Jacques) constituaient l'essentiel du roman. Diderot prend même à parti le lecteur en interrompant le récit, établissant un rapport subtil entre la liberté de l'écriture et le thème du fatalisme. Un conte de Noël se place dans cette perspective en disposant, en plus d'Elizabeth, deux autres personnages (Junon et Sylvia) qui prendront en charge à leur tour le récit de façon directe. En effet, Desplechin filme, à la mi-temps du récit et plein cadre, Junon chez elle. Elle raconte en in 
l'histoire de sa maison et de sa famille. Elle organise l'espace en faisant visiter son salon au spectateur puis clôt son récit en disant : "Grâce à ma maladie, nous serons tous réunis à Noël». Le procédé choisi est de l'ordre duredoublement du regard en miroir manifestant une mise en abyme de la fiction, voire même d'un personnage qui devient son propre partenaire. La voix-off n'est pas de la partie mais cet exemple insiste sur le fait que tout le récit appartient aux personnages. Le film est donc une fiction qui joue à l'autofiction tant par ces adresses directes à la caméra, qui sont des soliloques intimes, que par la voix-off actorielle. Par ailleurs, ces formes diverses de monologues annulent l'intervalle entre la fiction et la salle étant une invite à la fiction. Les multiples voix narratrices devenant des ponts entre deux rives narratives.

11 Par ailleurs, cette voix intérieure présente dans les voix-off actorielles ou les adresses à la caméra renvoient à la "moving box ${ }^{1}$ ", terme propre au cinéaste Terence Malick qui désigne métaphoriquement l'âme humaine. La voie narrative nous entraîne dans le for intérieur du protagoniste, dans les méandres de sa pensée. Cette voix intérieure est "décalée » car elle ne s'effectue pas dans un rapport linéaire avec le cours du récit. Le caractère autobiographique et autofictionnel, comme le côté théâtral des apartés, sont rejoints par la voix narrative de Nora (Rois et Reine). En racontant sa vie, elle emploie un présent intemporel. Si le dispositif est dissemblable entre les deux cinéastes, leur fonction est préservée car les voix internes isolent les personnages les uns des autres. D'autre part, chez Desplechin seule la voix narrative externe se mêle à l'air ambiant commentant les actions et dires des personnages à l'écran. Les autres voix se heurtent au mur de la conscience. Aussi, lorsque Elizabeth, Junon ou Sylvia s'expriment, la sensation est, qu'en dépit de s'adresser au spectateur, c'est aussi une porte qui s'ouvre et se ferme sur un obscur intérieur. Le spectateur est comme rejeté de leur univers figurant une insularité humaine. Le lien n'opérant ici que pour réunir les îlots de pensées. Dans une autre séquence d'Un conte de Noël, Elizabeth s'isole dans sa chambre. Elle regarde sa maison de poupée alors que sa voix-off résonne. Elle s'adresse à son frère Henri, celui qu'elle a banni. Elle dit le chercher comme son regard qui se perd dans les recoins de la maquette. Puis, le temps d'un plan, elle lui parle à voix haute. Enfin, en raccord cut, assise sur son lit face caméra, elle s'adresse toujours à lui et avoue ce qu'elle a enfin compris : « La maladie, c'était toi (...) Tu as volé ma vie entière ». Le plan regard caméra exprime le désarroi d'Elizabeth. Dans un premier temps, nous le comprenons comme étant l'aparté d'un comédien qui livre ses sentiments, ses secrets le temps d'une pause au sens d'immobilité physique et de suspension du récit initial. C'est une confession qui interrompt la fiction avant de reprendre son cours. Mais cette voix intérieure en in et en off exprime également son désir inaccessible de parfaire le monde qui l'entoure en le recréant et dévoile en cela la force des mots.

Prononcés dans l'intimité, ils cassent le relationnel sauvage et maladroit d'Elizabeth autant qu'ils soulignent sa maitrise plénière à cet instant de sa vie. La vacuité existentielle, le chagrin (elle cherche perpétuellement qui est mort), le sentiment de rejet et de frustration sont ici contenus dans ses mots sortis tout droit de sa " moving box ». Il faut se mettre en posture propre à pouvoir refléter sa conscience pour accéder à soimême. Ce regard caméra est donc l'expérience de déclencher l'écho de leurs mots et la réverbération de leur image pour se faire face et saisir leur réflexion. Au travers d'une structure close (effet miroir/isolement), il est peut-être possible de trouver plus facilement le sens à soi et au monde, échappant encore à l'altérité ou au rejet. Croire que 
les mots entendus nous révèlent. Si cela n'est pas une voix-off de bout en bout, cette adresse s'y substitue de par l'objet même, la confession.

Outre le texte littéraire en tant qu'inspiration du récit, le texte visible et/ou audible comme composante de celui-ci est à considérer. Les récits de Desplechin ne sont pas basés en entier sur des journaux intimes ou des lettres prononcés par la voix-off du héros comme pour Journal d'un curé de campagne (1951), Un condamné à mort s'est échappé (1956) ou bien Pickpocket (1959) de Bresson, voire certains films de Truffaut. Néanmoins, la lettre de Louis dans Rois et Reine dont nous percevons quelques mots écrits mais jamais sa transcription, et que Nora découvre dès les premières pages du manuscrit, est un exemple pertinent. Nora entre dans la chambre de son père mort quelques jours auparavant. Avec sa sœur Chloé, elle prépare le déménagement. Après qu'elle ait posé le regard sur un manuscrit placé à même le lit, un plan en raccord-cut s'ouvre sur une image étrange de son père face caméra. Il est assis sur un tabouret avec en fond une toile grise comme s'il était chez un photographe. Le grain de l'image est épais et proche des films anciens, sautant légèrement. Louis est tout d'abord cadré en plan moyen, puis la caméra zoome tout au long de la séquence et termine en plan rapproché. Il récite cette lettre de mémoire avec, en alternance, sa voix en off lorsque Nora lit mentalement la lettre, puis en prise directe lorsqu'il est à l'écran. Le passage en direct ne l'est seulement qu'à l'endroit du spectateur. La lecture de la lettre recouvre les plans au présent mais convoque aussi des images issues d'un souvenir déjà vu auparavant. Desplechin refuse de montrer l'écriture comme pour insister sur le besoin de déceler les émotions sur le visage et le corps du personnage alors que se distille le pouvoir des mots paternels. Lorsqu'il prononce : "Tu es aigre et ta vanité est devenue une coquetterie », Nora devient pâle et ôte l'élastique qui retenait ses cheveux comme atteinte au plus profond d'elle-même. Elle annule ce qui la rend féminine et apprêtée. Au lieu de priver le spectateur de ce détail, Desplechin choisit de le livrer creusant à nouveau le récit et le rendant plus proche du romanesque littéraire jusque dans son appréhension psychique et mentale. D'autre part, approchant timidement de la stratégie bressonienne du doublage de l'événement dû aux textes intérieurs au film, on ne peut jamais savoir chez Desplechin si ce qu'énonce la voixoff est ce qui est écrit, mais la coexistence de la base littéraire et de l'image est une façon originale d'intensifier l'image. Louis avait l'habitude de réciter des poèmes à sa fille, aussi sa voix a-t-elle une importance. Les images brèves du flash-back sont ces moments de complicité et de poésie récitée. Quant à la voix du père, elle se "perpétue " d'entre les morts comme l'indique l'intertitre de ce paragraphe du récit. Plus tard, Nora se marie et, au moment de brûler cette lettre, plusieurs plans de Louis apparaissent. Identiques aux premiers plans cités, ceux-ci semblent avoir été en suspens comme en attente d'une réponse de la part de Nora. À mesure que les flammes racornissent le papier, le père se recroqueville en gémissant. Nora face caméra imagine cette scène, qui n'émane que de sa conscience. Dans Un conte de Noël, Desplechin reprend quasiment le même dispositif que pour Louis en disposant Henri sur un siège face caméra avec en fond visuel une toile grise. Mais le mouvement de caméra qui rapproche le spectateur du scripteur visualisé est cette fois-ci démesuré. En effet, la caméra arrive au cœur de sa pupille.

Identifiant la lettre au personnage et signifiant métaphoriquement un voyage au centre de son cerveau comme le plan au cœur de la tête Jivaro de La Sentinelle, nous sommes entrainés vers son esprit "malade", dixit sa sœur. Toutefois, cette figure peut aussi se comprendre comme une incapacité à prendre du recul envers les mots d'Henri. Vérité ou manipulation, sous couvert d'une prose adéquate se cachent en fait quelques sentiments 
«diaboliques ». Ainsi le son, le mot prononcé, importe plus que l'écriture doublée de la voix-off. Il y a dématérialisation par é-vocation. Ce matériau sonore permet de faire surgir l'Idée au sens platonicien du terme, ainsi que de produire des images fugaces et permanentes. En cela, la lecture à voix haute d'Esther de sa lettre à Paul devient une sorte d'incantation dans Comment je me suis disputé (ma vie sexuelle). Écho parfait à celle de Muriel (Les Deux anglaises et le continent de Truffaut) qui, parcourant la lande puis de retour chez elle, face caméra, exprime son amour à claude. Toutes deux cherchent à faire entendre ce qu'elles ne savent dire, voire peut-être ce qu'elles ignoraient jusqu'à leur formulation. Le regard caméra, dans ces deux exemples, est un redoublement de la pensée par une image sensible qui la fixe, puis son expression par un mot ou un discours pour la faire sortir de notre intériorité. Car, étrangers à nous-mêmes, c'est bien dans notre intériorité que s'ébauche l'amorce d'un dialogue intérieur.

En conséquence, nous pouvons affirmer que cette structure narrative propre à Desplechin multistratifiée organise pour une part l'opacité du discours comme si le cinéaste poussait paradoxalement le spectateur à croire en l'image pour combattre le scepticisme que le sonore ne permet pas vraiment puisque rien n'établit avec certitude la vraisemblance. Par ailleurs, les monologues des voix narratrices forment des îlots de mots trahissant le désir d'une coulée sonore continue, organisant les rythmes et liant les pulsions de vie au-delà d'une simple juxtaposition sémantique et formelle. Enfin, en plus de l'adresse caméra d'Elizabeth (Un Conte de Noël), le dernier plan la montre en train de rédiger un journal. Un contre-champ dévoile sur le mur son ombre chinoise écrivant tandis que sa voix-off accompagne les images. Elle le voit, en joue et sourit tout en reposant le crayon. Desplechin cherche au travers de la transmission des discours à faire émerger les émotions, les sensations, les pensées, ce qui en somme n'arrive pas à poindre lorsque l'Autre fait face. Aussi, la rédaction du journal d'Elizabeth est-elle également l'illustration parfaite d'un cogito qui se saisit en s'exprimant (et en se reflétant). Elle y annote ce qu'elle a appris et compris. C'est un travail introspectif et réflexif. La conscience entre les lignes. L'ultime plan la montre sur le balcon de son appartement. Sa voix-off annonce que Junon va guérir car la greffe va prendre et qu'elle, Elizabeth, vit désormais dans le pays inventé par son fils. Elle conclut par une libre adaptation de l'épilogue du Songe d'une nuit d'été de Shakespeare : «Ombres que nous sommes, si vous avez déplu, pensez ceci : vous n'avez fait que dormir, tout ceci n'était qu'un rêve et tout sera réparé ». Étalés dans l'espace, les mots prononcés deviennent maîtrisables, ce qui est symptomatique des personnages chez Desplechin. De la même manière que parler, dire le monde, c'est le vivre et non plus le subir. Que le discours soit oralisé, visualisé, transcrit, la conscience est toujours ce qui se parle. Il n'y a pas de vie humaine, de prise de conscience, sans langage.

\section{ANNEXES}

Marie-Anne Lieb

Domaines de recherches : Narration, Cinéma français contemporain, Esthétique, Contemplation, Enfance. 
Publications aux Presses Universitaires de Caen- Double jeu :

$\mathrm{N}^{\circ}$ 7- 2011, «Exprimer l'échancrure affective au moyen d'une forme pleine. De Private fears in public places d'Alan Ayckbourn à Cours d'Alain Resnais ", in Resnais et le théâtre $\mathrm{N}^{\circ}$ 6- 2009, PUC, « Le huitième climat ou l'ailleurs contemplatif dans le cinéma de N.B.Ceylan», inAction/Contemplation

$\mathrm{N}^{\circ} 4$-2007, PUC, « Entre Marivaudage et analyse- Le discours amoureux chez Desplechin comme quête de soi », in Discours amoureux, scènes de séduction.

Journée d'étude : Filmer la création- le jeu de l'acteur en question, « Desplechin et les acteurs : pratique corporelle, gestuelle et vocale ou un jeu de tempo ", à l'initiative des universités de Rennes 2, de Caen et de l'ANR. Publication à venir aux PUR.

Colloque : « Voix-off et narration cinématographique », Nice- Octobre 2011.

\section{NOTES}

1. Michel Chion, La ligne rouge, BFI, 2004, Les Editions de La Transparence/ Cinéphilie, 2005.

\section{RÉSUMÉS}

L'œuvre d'Arnaud Desplechin est particulièrement intéressante à bien des égards et a fortiori pour sa virtuosité narrative. Cinéma romanesque et introspectif, la narration passe entre autres, par l'usage savant de la voix-off comme mode d'énonciation ou par celui des voix (es) narratives proches de la «moving box" propre à Terence Malick. Des aventures spirituelles, au marivaudage et aux quêtes existentielles, leur utilisation au sein de la filmographie confère aux personnages une tendance à l'affabulation. L'enjeu réside en ce que le cinéaste cherche à raconter une histoire autant que ses personnages tentent de se raconter eux-mêmes. Recomposant la complexité humaine, Desplechin déploie un cinéma dans lequel la voix-off ouvrent un peu plus les possibles narratifs en forme d'insularité humaine.

Arnaud Desplechin's work is particularly interesting in many respects but especially so for his narrative virtuosity. In his style of cinema, which combines both story-telling and introspection, the plot unfolds by the wise use, amongst other things, of voice-over as a means of statement or by narrative voices similar to the "moving box" technique used by Terence Malick. The use of such techniques, ranging from spiritual adventures to light hearted banter and existential quests, in the heart of filmography can give the impression that characters do not ring true. For the film-maker, the problem is how to balance the unfolding of the overall story-line with the exteriorisation of each character's personal thoughts. By rearranging human complexity, Desplechin develops a cinema in which the voice-over progressively opens up the possible narratives reflecting human insularity. 
INDEX

Index géographique : France

Mots-clés : conscience, dédoublement, fabulation, introspection, moving box, voix intérieure Index chronologique : XIXème, XXème, XXIème

\section{AUTEUR}

\section{MARIE-ANNE LIEB}

Université de Caen. (Université, centre de recherche et éventuellement, principaux travaux ou domaines de recherche) : Docteur en Etudes cinématographiques- Université de Caen /BasseNormandie. Enseignante vacataire à l'Ecole de Management de Caen et à l'Université Catholique de l'Ouest à l'Institut des Arts, Lettres et Histoire. Formatrice « Collège au cinéma » depuis 2004. 